\title{
Failure Modes of Fluid Cell Chambers Used for Liquid In Situ Transmission Electron Microscopy
}

\author{
E. R. White, William A. Hubbard, and B. C. Regan \\ Department of Physics \& Astronomy and California NanoSystems Institute, University of California, \\ Los Angeles, California 90095 USA
}

The ability to image liquid samples with a transmission electron microscope (TEM) is a recent advance; only within the last decade have the difficulties in preparing a TEM-ready liquid sample been overcome using modern nanofabrication techniques [1]. A new field where TEM experiments can be done with liquids in situ has since grown rapidly [2]. Still, with these new fabrication techniques building a working fluid cell is not a trivial endeavor. Here we will discuss some of the failure modes and other difficulties encountered while performing in situ liquid TEM.

To image a high vapor-pressure liquid in a TEM, the liquid must be isolated from microscope's high vacuum. A typical fluid chamber consists of two aligned, electron-transparent windows in silicon chips sandwiching a thin layer of liquid[1-3]. Some designs allow for fluid exchange in situ, while others are sealed. In both cases the internal cell pressure is typically (though not always) one atmosphere. Thus when the fluid cell is put into the microscope's high vacuum the pressure differential across a membrane causes it to bow into the vacuum. If the membranes are too weak to support the stress they rupture. Figure 1 shows before-and-after optical pictures of a fluid cell that burst when placed into an evacuated chamber. The cell was filled with an aqueous solution of cesium chloride which crystallized after the water evaporated.

The fluid layer must be thin, typically $1 \mu \mathrm{m}$ or less, to achieve high resolution. While assembling our sealed fluid cells, we apply force to the top silicon chip while sealing the chamber. The force applied represents a delicate balance between achieving a thin liquid layer and risking membrane rupture or deformation.

The use of certain solutions can affect the shelf-life of a sealed fluid cell. Figure 2 shows two optical photographs of a fluid cell containing uranyl chloride. A graphene sheet is visible between two gold electrodes. Figures $2 \mathrm{a}$ and $2 \mathrm{~b}$ show that the graphene sheet changes over a three day period - the cesium chloride apparently attacks the graphene. Figure 2c, a scanning TEM (STEM) picture of the graphene sheet several days after the fluid cell was sealed, shows evidence of this chemical attack.

Contamination can be a major problem with in situ liquid TEM studies. Care must be taken to ensure that the sample, the sample holder, and microscope column are all free of volatile hydrocarbons which would be deposited on the sample by the beam. Low-outgassing epoxies must be used for sealing cells. Buildup of contamination on the sample decreases resolution and complicates any quantitative analysis done on times series of images.

Understanding the different failure modes of the TEM fluid cell chamber is critical for consistently producing quality samples. Proper thickness of the membrane windows and liquid layer, chemical effects of the cell solution on other cell components, and contamination are all important considerations when building a fluid cell chamber. 
References:

[1] M. J. Williamson et al., Nature Materials 2 (2003), p. 532.

[2] N. de Jonge and F. M. Ross, Nature Nanotechnology, 6 (2011), p. 695.

[3] E. R. White et al., Applied Physic Express 4 (2011), p. 055201.

[4] E. R. White et al., ACS Nano 6 (2012), p. 6308.

[5] This work was supported by the American Chemical Society's Petroleum Research Fund, NSF grants DMR-0748880 and DMR-1206849, and the Electron Imaging Center for NanoMachines at UCLA.
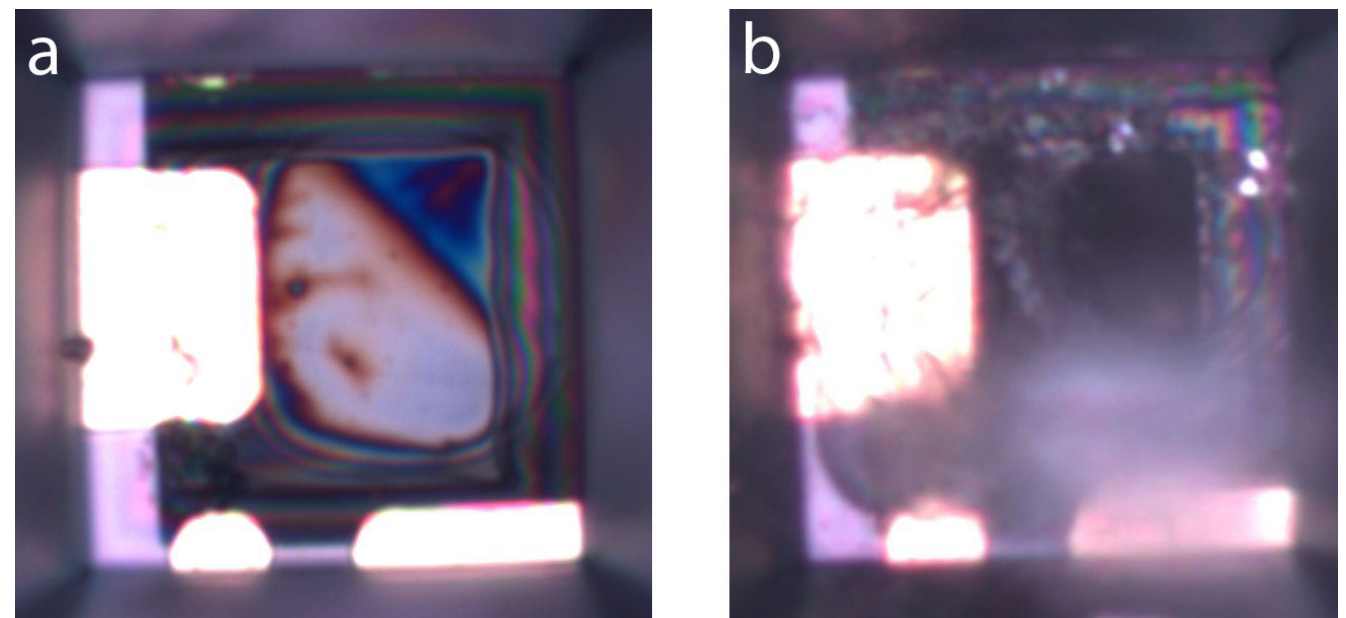

Figure 1. Optical photographs of the membrane windows of a completed fluid cell. The pictures are taken with a 50x, NA=0.55 objective. Each window is approximately $30 \mu \mathrm{m}$ on a side. (a) Completed fluid cell immediately after construction. The silicon nitride windows are separated by a thin layer of water saturated with $\mathrm{CsCl}$. Gold contacts and alignments markers are also visible. (b) The same fluid cell after placing it in vacuum. The windows have ruptured, the water has evaporated, and the $\mathrm{CsCl}$ has crystallized in towers that extend many micrometers out of the focal plane.
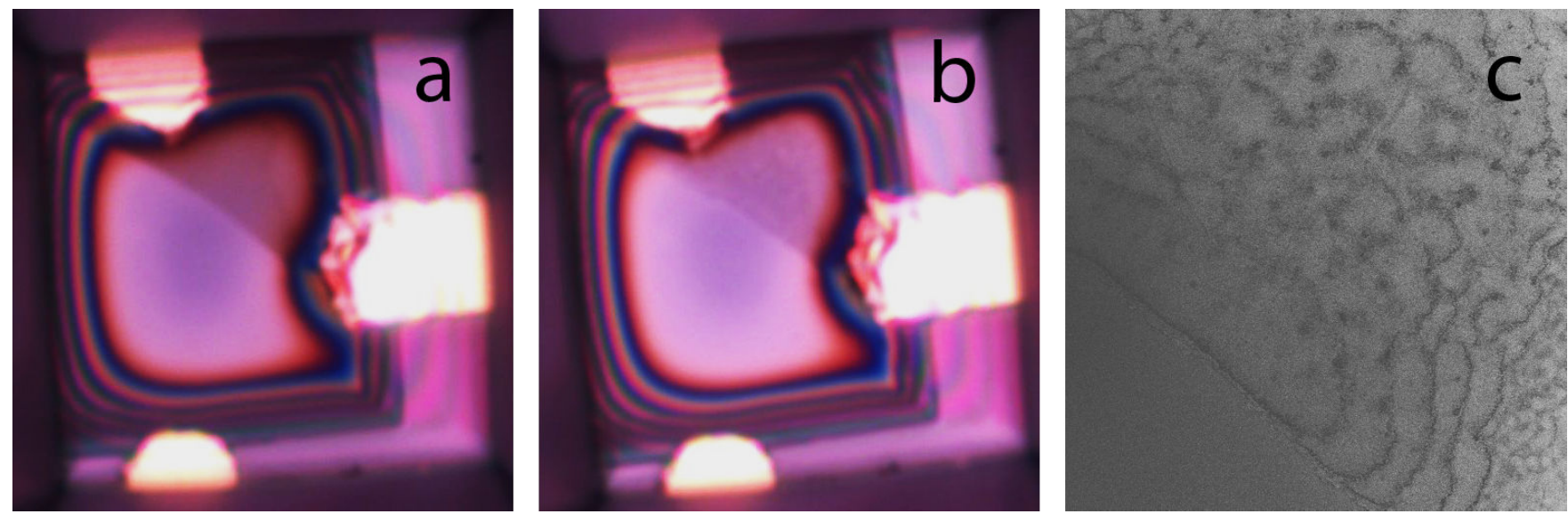

Figure 2. (a) Optical photograph of the membrane windows of a completed fluid cell filled with a saturated solution of uranyl chloride immediately after construction. A sheet of graphene connects the gold electrodes entering from the top and right edges of the photograph. (b) The same fluid cell three days later shows that the graphene has been altered. (c) STEM image of the graphene sheet after the photograph in (b) was captured. 